Accepted after revision: September 23, 2008

\title{
Differential FGF Ligands and FGF Receptors Expression Pattern in Frontal and Parietal Calvarial Bones
}

\author{
Natalina Quarto ${ }^{a}$ b Björn Behr ${ }^{a}$ Shuli Li ${ }^{a} \quad$ Michael T. Longaker ${ }^{a}$ \\ a Children's Surgical Research Program, Department of Surgery, Stanford University School of Medicine, \\ Stanford, Calif., USA; ${ }^{b}$ Department of Structural and Functional Biology, University of Naples Federico II, \\ Complesso M.S. Angelo, Napoli, Italy
}

\section{Key Words}

Neural crest $\cdot$ Mesoderm $\cdot$ Origin $\cdot$ Frontal $\cdot$ Parietal $\cdot$ Bone $\cdot$

FGF/FGFR • Mouse

\begin{abstract}
The mammalian skull vault consists mainly of 5 flat bones, the paired frontals and parietals, and the unpaired interparietal. All of these bones are formed by intramembranous ossification within a layer of mesenchyme, the skeletogenic membrane, located between the dermal mesenchyme and the meninges surrounding the brain. While the frontal bones are of neural crest in origin, the parietal bones arise from mesoderm. The present study is a characterization of frontal and parietal bones at their molecular level, aiming to highlight distinct differences between the neural crest-derived frontal and mesodermal-derived parietal bone. We performed a detailed comparative gene expression profile of FGF ligands and their receptors known to play crucial role in skeletogenesis. This analysis revealed that a differential expression pattern of the major FGF osteogenic molecules and their receptors exists between the neural crest-derived frontal bone and the paraxial mesoderm-derived parietal bone. Particularly, the expression of ligands such as Fgf-2, Fgf-9 and Fgf-18 was upregulated in frontal bone on embryonic day 17.5, postnatal day 1 and postnatal day 60 mice. Frontal bone also elaborated higher levels of Fgf receptor 1, 2 and 3 tran-
\end{abstract}

scripts versus parietal bone. Taken together, these data suggest that the frontal bone is a domain with higher FGF-signaling competence than parietal bone.

Copyright $\odot 2009$ S. Karger AG, Basel

\section{Introduction}

The mammalian skull is the product of an evolutionary process during which 4 skeletal components of independent origin have progressively integrated into a structure of exquisite structural and functional complexity [Morriss-Kay, 2001]. The 4 components of the vertebrate skull are the cartilaginous neurocranium, cartilaginous

\begin{tabular}{ll}
\hline Abbreviations used in this paper \\
\hline BrdU & $\begin{array}{l}\text { bromodeoxyuridine } \\
\text { embryonic }\end{array}$ \\
E & fibroblast growth factor \\
FGF & fibroblast growth factor receptor \\
FGFR & glyceraldehyde 3-phosphate dehydrogenase \\
Gapdh & proliferating cellular nuclear antigen \\
PCNA & postnatal \\
pN & runt-related transcription factor 2 \\
Runx2 & \\
RT-PCR &
\end{tabular}

Tel. +1 650736 1704, Fax +1 650736 1705, E-Mail quarto@unina.it 
viscerocranium, dermal skull roof and the sclerotomal occipital region.

The vertebrate dermal skull roof is an ancient structure formed from membrane bones that are evolutionarily derived from the protective dermal plates of early jawless fishes. The mammalian skull vault is constructed principally from 5 bones: the paired frontals and parietals, and the unpaired interparietal. These calvarial bones arise from 2 tissues, neural crest and mesoderm. The distinct contributions of each tissue to the skull have been well established by combining mice with a Wnt1-Cre construct and a conditional reporter gene, $R 26 R$ [Chai et al., 2000; Jiang et al., 2002]. These studies have defined the pattern of cranial neural crest cell migration in mouse embryos and demonstrated that the frontal and squamosal bones are of neural crest origin, in contrast to the parietal and interparietal bone, which are of mesoderm origin; the unossified sutural mesenchyme between the parietal bones is also of neural crest origin.

Development of the normal skull vault requires mechanisms to ensure that both its morphology and its rate of growth are precisely matched to those of the developing brain. This precise relationship suggests that there are important tissue interactions between the brain and the skeletogenic membranes also involving the mesenchymal layers between them (the developing meninges).

A multitude of signaling molecules, as well as their respective receptors and downstream transcriptional factors, play in concert to regulate bone development [Karsenty, 2001; Karsenty and Wagner, 2002]. In particular, fibroblast growth factor (FGF) signaling has gained much attention for its major role in skeletogenesis, including calvarial osteogenesis [Muenke and Schell, 1995; Naski and Ornitz, 1998; Ornitz and Itoh, 2001; Marie, 2003]. FGF signaling is known to play a critical role in regulating proliferation and differentiation of osteoblasts and osteogenic precursors [Mansukhani et al., 2000; Kim et al., 2003; Marie, 2003; Fakhry et al., 2005; Quarto and Longaker, 2006]. Its role in bone biology has been highlighted by the skeletal phenotypes of the numerous FGF ligand and receptor transgenic mice [Coffin et al., 1995; Deng et al., 1996; Eswarakumar et al., 2002; Liu et al., 2002; Ohbayashi et al., 2002; Govindarajan and Overbeek, 2006; Hung et al., 2007]. Thus, delineating regional differences in osteogenic potential based on embryonic origin and determining the role of FGF signaling in imparting superior regenerative potential on calvarial tissue is of paramount importance. However, little has been done to investigate whether regional differences in the expression of signaling molecules occurs between frontal and parietal bones.
Indeed, obtaining a comprehensive understanding of unique cell biology and gene expression patterns of calvarial osteoblasts from dissimilar embryonic origins, and how those differences translate into variable potential for endogenous bone regeneration, will be critical to the optimization of cell-based skeletal tissue engineering strategies.

In this study, we have performed a detailed comparative gene expression profile of FGF ligands known to play crucial roles in skeletogenesis and their receptors. The analysis presented here reveals that a differential expression pattern of the major FGF osteogenic molecules and their receptors exists between the neural crest-derived frontal bone and the paraxial mesoderm-derived parietal bone. Particularly, the expression of ligands such Fgf-2, $\mathrm{Fgf-} 9$ and $\mathrm{Fgf-18}$ is upregulated in frontal bone in embryonic day 17.5 (E17.5), postnatal day 1 (pN1) and postnatal day 60 (pN60) mice. Frontal bone also elaborated higher levels of $F g f$ receptors $F g f R 1, F g f R 2$ and $F g f R 3$ transcripts versus parietal bone. Taken together, these results suggest that the neural crest-derived frontal bone is a highly activated FGF-signaling domain compared to parietal bone.

Thus, this gene profiling study begins to establish molecular differences between these 2 calvarial bones of different tissue origin. Moreover, the data indicate that differences in tissue origin may dictate a distinct FGF-signaling pattern between frontal and parietal bones.

\section{Materials and Methods}

\section{Animals}

All experiments using animals were performed in accordance with Stanford University Animal Care and Use Committee guidelines. CD-1 wild-type mice were purchased from Charles River Laboratories Inc. (Wilmington, Mass., USA). At birth, animals were considered 0.5 days old. Animals were housed in light- and temperature-controlled rooms and were given food and water ad libitum. After euthanizing the animals, skulls of E17.5, pN1 and pN60 mice were isolated using a stereo microscope.

\section{Tissue Staining}

Following sacrifice of animals as described above, the skulls were harvested and immediately fixed in fresh, chilled $4 \%$ phosphate-buffered paraformaldehyde overnight at $4{ }^{\circ} \mathrm{C}$ followed by washing in phosphate-buffered saline-0.1\% Tween-20 for 30 min.

For bony tissue detection, specimens were stained with $0.1 \%$ alizarin red and $3 \%$ potassium hydroxide overnight at room temperature. All specimens were cleared in $50 \%$ glycerol and $5 \%$ potassium hydroxide for 2-7 days. Specimens were analyzed under a dissecting microscope. Images were acquired using a Leica MZ16 equipped with a DC500 digital camera. 
Table 1. Nucleotide sequence of primers and annealing temperature used for PCR analysis

\begin{tabular}{llll}
\hline Gene & Forward primer & Reverse primer & $\begin{array}{l}\text { Annealing } \\
\text { temperature, }{ }^{\circ} \mathrm{C}\end{array}$ \\
\hline$F g f-2$ & CGGCATCACCTCGCTTCC & CTTCTGTCCAGGTCCCGTTTTG & 58.6 \\
$F g f-9$ & TCGCCTAGTGTCTCCTGGTTGATA & CCATACAGCTCCCCTTCTCGTTC & 58.7 \\
$F g f-18$ & TGGCAGCCGAGGAGAATGTGGACT & CCGCCGGGATCGCTTGGTGAC & 61.7 \\
$F g f R 1$ & GTGGCCGTGAAGATGTTGAAGTCC & GCCGGCCGTTGGTGGTTT & 59.1 \\
$F g f R 2 b$ & CACCCGGGGATAAATAGCTCCAATG & GCTGTTTGGCAGGACAGT & 60.0 \\
$F g f R 2 c$ & CACCCCGGTGTTAACACCACGG & CTGGCAGAACTGTCAACCATG & 60.0 \\
$F g f R 3$ & TGCCGGCCAACCAGACAGC & GCGCAGGCGGCAGAGTATCAC & 61.4 \\
\hline
\end{tabular}

\section{Tissue Harvest}

Fresh tissue was harvested from the frontal and parietal calvarial bones of E17.5, pN1 and pN60 mice (1 litter, on average 9 animals). The tissue was harvested from 3 independent littermates. After dissecting out the parietal and frontal bones meticulously to exclude dura mater, pericranium and suture-associated osteogenic fronts, the tissues were immediately snap frozen in liquid nitrogen. To extract RNA, the bone chips were mechanically homogenized in Trizol reagent using a pellet pestle motor (Kontes, Vineland, N.J., USA).

\section{RNA Isolation and RT-PCR Analysis}

Total RNA was isolated by using Trizol reagent according to the manufacturer's instructions (Invitrogen, Carlsbad, Calif., USA). Purified and quantified RNA was treated with DNAse I (Ambion, Austin, Tex., USA) to clear genomic DNA. Two micrograms of total RNA was then reverse transcribed to cDNA using random hexamer primers (Invitrogen). Reverse transcription was performed for $1 \mathrm{~h}$ at $42^{\circ} \mathrm{C}$, followed by incubation at $75^{\circ} \mathrm{C}$ for $5 \mathrm{~min}$ to inactivate reverse transcriptase. RNAse treatment was used to clear residual RNA. PCR were performed under the following conditions: $94^{\circ} \mathrm{C}$ for $5 \mathrm{~min}, 94^{\circ} \mathrm{C}$ for $30 \mathrm{~s}$, annealing for $1 \mathrm{~min}$ (see table 1 for the annealing temperature), and $72^{\circ} \mathrm{C}$ for $1 \mathrm{~min}$ (25-30 cycles). Specific primers for the genes examined were designed based on their GenBank sequence. Primer sequence and PCR conditions for the Gapdh and Runx2 (Cbfa-1) genes have been previously described [Quarto and Longaker, 2006]. Primer sequences for the other genes are listed in table 1. For the densitometry analysis of RT-PCR, bands were scanned and quantified by using the Image program 1.36b (National Institutes of Health, Bethesda, Md., USA). The densitometry results of each band were normalized to their respective loading control (Gapdh) and presented as percentage increase. The scanning was performed on 3 independent experiments (3 different littermates) and results are presented as means \pm SE of the 3 independent experiments. Quantitative RT-PCR was performed as previously described [Quarto et al., 2005]. The results are presented as means \pm SE of 2 independent experiments (online suppl. fig. 1, www.karger.com/doi/10.1159/000202789).

\section{Statistical Analysis}

The results are presented as means \pm SD of 2 or 3 independent experiments performed on different littermates. Statistical differences between the means were examined by Student's t test. $p<$ 0.05 was considered statistically significant.

\section{Frontal and Parietal Primary Osteoblast Culture}

Calvarial osteoblasts were harvested from the frontal and parietal bones of E17.5, pN1 and pN60 mice. Briefly, the frontal and parietal bones were removed under direct visualization using a dissecting microscope, taking great care to exclude suture-associated bone. The periosteum and dura mater were meticulously removed from the calvaria, and only nonsuture-associated frontal and parietal bone was dissected free from surrounding tissue. Calvarial bones were subsequently mechanically minced and subjected to enzymatic digestion in serum-free media. Frontal and parietal bones depleted of periosteum, suture mesenchyme and dura mater were digested with $0.2 \%$ dispase II and $0.1 \%$ collagenase A (Roche Diagnostics, Indianapolis, Ind., USA) in serum-free medium. The digestion was repeated 6 times for $10 \mathrm{~min}$ each for frontal and parietal bones from E17.5 and pN1 mice and 15 min each for $\mathrm{pN} 60$ mice, at $37^{\circ} \mathrm{C}$ in a water bath shaker. The first 2 digestions were discarded. The latter 4 digestions were pooled, pelleted and resuspended in $\alpha$-minimal essential medium $(\alpha$-MEM) supplemented with $10 \%$ FCS. First-passage osteoblasts were plated at equal density and cultured to subconfluence in growth media ( $\alpha$-MEM, 10\% FCS, $100 \mathrm{IU} / \mathrm{ml}$ penicillin and streptomycin).

\section{Cell Proliferation Assays}

The growth rates of frontal and parietal calvarial osteoblasts were assessed by BrdU labeling assay. Frontal and parietal osteoblasts (at passage 1) were plated at 1,500 cells/well, in 96-multiwell culture plates with flat bottoms (Corning, New York, N.Y., USA). Cells were washed twice with sterile phosphate-buffered saline and starved in serum-free $\alpha$-MEM overnight. BrdU incorporation was carried out for $24 \mathrm{~h}$ (Roche Diagnostics) according to the manufacturer's instructions. Photometric detection was done with an ELISA reader at $370 \mathrm{~nm}$ wavelength. The background was subtracted when the resulting data were processed. Each time point was run in triplicate.

\section{Immunohistochemistry}

Mouse calvaria were harvested at pN7 and pN60. Samples were fixed in $10 \%$ buffered formalin overnight at $4^{\circ} \mathrm{C}$, decalcified, processed for paraffin embedding and cut in $10-\mu \mathrm{m}$ sections. Selected paraffin sections were chosen and heat antigen retrieval was performed. For proliferating cell nuclear antigen (PCNA) staining, the PCNA staining kit was used according to the manufacturer's instructions (Invitrogen). Biotinylated secondary 

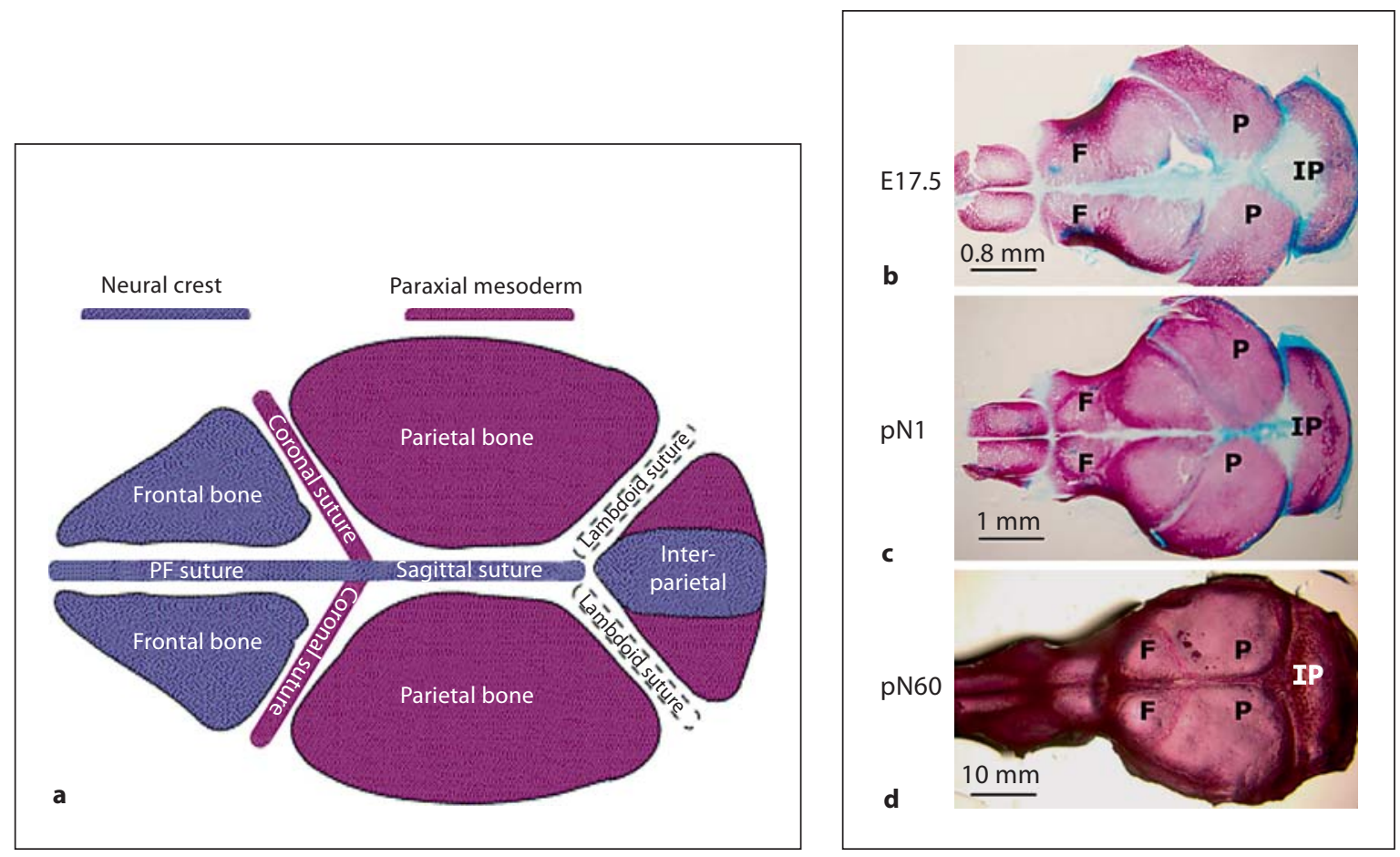

Fig. 1. Schematic depicting neural crest and mesoderm origin of cranial vault. a Anatomy of skull vault (bones and sutures) and the neural and paraxial mesoderm origin of calvarial bones. Alizarin red staining of skull vault derived from E17.5 (b), pN1 (c) and pN60 (d) mice assesses the extent of bone mineralization. PF = Parietofrontal; IP = interparietal.

anti-rabbit antibody was used at 1:200 dilutions. Immunostaining with normal (irrelevant) rabbit IgG (sc-2027; , Santa Cruz Biotechnology Inc., Santa Cruz, Calif., USA) did not detect any staining (data not shown). The slides were analyzed and photographed with a Zeiss Axioplan 2 microscope equipped with an Axiocam HRc digital camera.

\section{Results}

\section{Osteogenesis of Skull Vault}

The mammalian skull vault consists of 5 bones that mineralized directly in membrane: a pair of frontal bones, a pair of parietal bones and an interparietal bone; the interparietal bone later fuses with the endochondral component of the occipital bone to became the superior part of that bone. Figure 1a illustrates schematically the anatomy of skull vault and the neural and mesodermal origin of calvarial bones.

Alizarin red staining (fig. 1b) shows the mineralizing pattern of skull vault at E17.5. By E17.5, the frontal and parietal bones are mineralized in the lateral part of their future domains; interparietal mineralization can be ob- served as a narrow transverse line at some distance caudal to the parietal bones. Mineralization starts from the lateral areas. By pN1, mineralization of frontal and parietal bone is further progressed, both pairs of bone are almost completely stained with alizarin red (fig. 1c). Intense uniform staining is observed in pN60 skull vault, as a result of a completed mineralization (fig. 1d).

\section{Expression of FGF Ligands in Embryonic Skull Vault}

As an initial approach to define potential molecular and biological differences between the 2 calvarial bones of different tissue origin, we analyzed the gene expression profile of FGF ligands known to play a major role in osteogenesis. RT-PCR was performed on fresh tissues harvested from embryos at E17.5. In order to analyze the endogenous expression of osteoblasts, tissues were depleted of dura mater and pericranium. We found substantially elevated levels of Fgf-2, Fgf-9 and Fgf-18 gene expression in the frontal bone relative to the parietal bone (fig. 2a-c). Under these PCR experimental conditions we did not detect any expression of $F g f-4$ and $F g f-8$ either in frontal or parietal bone (data not shown). Because FGF ligands such 
Fig. 2. Gene expression profile of Fgf ligands in E17.5 frontal and parietal bones. a Fgf-2 expression was detected by RTPCR in tissue harvested from frontal and parietal bones. Fgf-2 transcript was strongly upregulated in frontal bone. b Fgf-9 expression was also increased in frontal bone, however, its transcript level was less abundant than Fgf-2 and -18. c Increased elaboration of $F g f-18$ transcript in frontal versus frontal bone was detected. $\mathbf{d}$ Frontal bone also expressed elevated levels of Runx2, a marker of osteoprogenitor cells. Histograms represent the densitometry analysis of RT-PCR products performed using the Image J software, the relative intensities of bands were normalized to their respective loading control and set as $100 \%$. -rt is a negative control for the reverse transcription reaction performed on pooled RNAs purified from each tissue. The results are presented as the means \pm $\mathrm{SD}$ of 3 independent experiments performed on different littermates. ${ }^{*} \mathrm{p}<0.01$. $\mathrm{F}=$ Frontal; $\mathrm{P}=$ parietal .

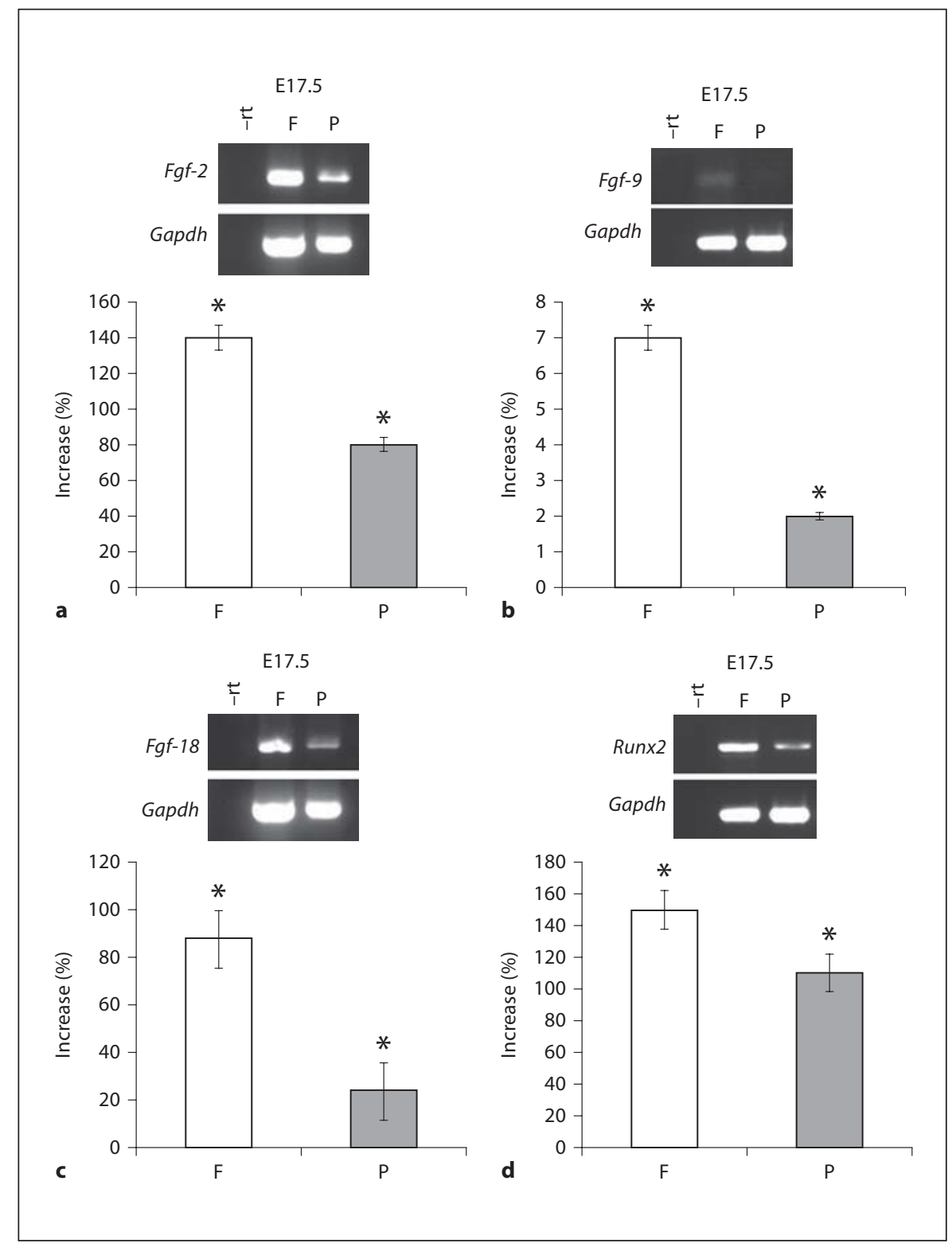

as Fgf-2 and Fgf-9 are known to trigger proliferation of osteoprogenitor cells [Fakhry et al., 2005; Quarto and Longaker, 2006], we investigated the expression level of Runx2, a marker of osteoprogenitor cells in frontal and parietal bones. Interestingly, embryonic frontal bone also elaborated higher levels of Runx2 (fig. 2d). The above results were further confirmed by quantitative realtime PCR analysis (online supplementary fig. 1, www. karger.com/doi/10.1159/000202789).

\section{Expression of FGF Ligands in Newborn Skull Vault}

Next, we analyzed the Fgf-2, Fgf-9 and Fgf-18 gene profiles in newborn mice. Fresh tissue was harvested from pN1 mice and RT-PCR was performed as above. We found that also in pN1 frontal bone the expression of all $3 \mathrm{Fgf}$ genes was higher compared to the parietal bone (fig. 3ac). However, overall, the levels of Fgf-2 and Fgf-9 transcripts in $\mathrm{pN} 1$ frontal bone were lower than that observed in embryonic frontal bone. Similarly to E17.5, Runx2 transcript was elevated in the frontal bone (fig. $3 \mathrm{~d}$ ). The above results were further confirmed by quantitative 
Fig. 3. Gene expression profile of Fgf ligands in $\mathrm{pN} 1$ frontal and parietal bones. a Increased levels of Fgf-2 transcript were detected in frontal bone compared to parietal bone. $\mathbf{b}$ RT-PCR analysis revealed only a faint expression of $\mathrm{Fgf}-9$ gene in frontal bone. Absence of Fgf-9 expression was observed in parietal bone. c Higher levels of Fgf-18 gene expression, similarly to that observed on E17.5, were detected in frontal bone versus parietal bone. d Runx2 was also elevated in frontal bone of $\mathrm{pN} 1$ mice. Histograms represent the densitometry analysis of RT-PCR products performed using the Image J software, the relative intensities of bands were normalized to their respective loading control and set as $100 \%$. -rt is a negative control for the reverse transcription reaction performed on pooled RNAs purified from each tissue. The results are presented as the means \pm SD of 3 independent experiments performed on different littermates. ${ }^{*} \mathrm{p}<0.01$. $\mathrm{F}=$ Frontal; $\mathrm{P}=$ parietal.
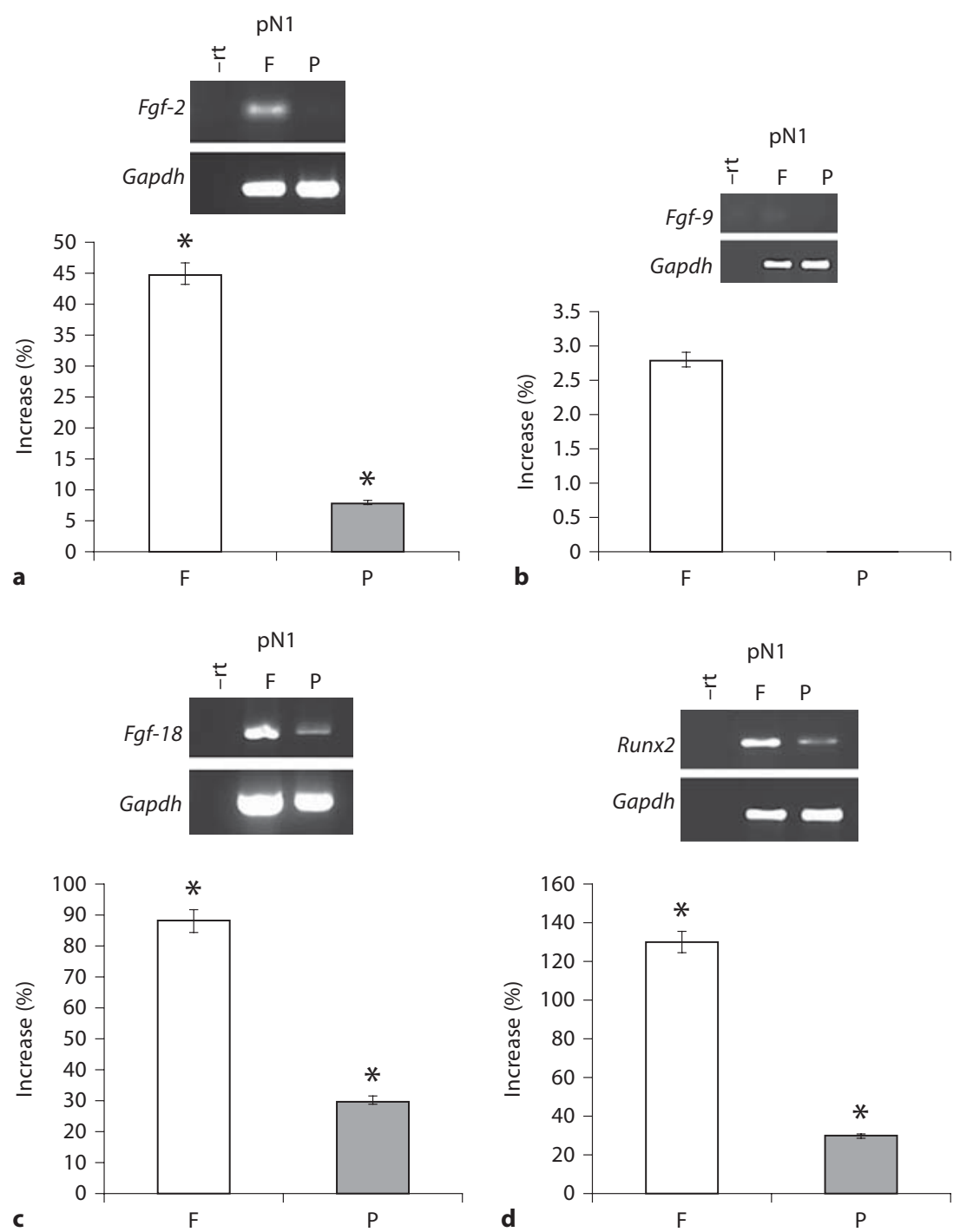

real-time PCR analysis (online supplementary fig. 1, www.karger.com/doi/10.1159/000202789).

\section{Expression of FGF Ligands in Adult Skull Vault}

The trend of Fgf-2 and Fgf-18 genes profile was maintained throughout adult organisms. We observed a quantitative differential expression pattern of $\mathrm{Fgf}-2$ and $\mathrm{Fgf}$ 18 , between adult frontal and parietal bones, with frontal bone having higher expression of $F g f-2$ and $F g f-18$ (fig. 4a, c). However, the threshold level of these genes was much lower than that observed either in embryonic or newborn mice. No Fgf-9 transcript was detected in pN60 frontal and parietal bones at least under these PCR experimental conditions (fig. 4b). Runx 2 gene expression was also upregulated in the frontal bone of adult mice (fig. $4 \mathrm{~d}$ ). The above results were further confirmed by quantitative real-time PCR analysis (online supplementary fig. 1, www.karger.com/doi/10.1159/000202789).

\section{Expression of FgfRs in Embryonic Skull Vault}

Because the initial study revealed that the neural crestderived frontal bone expressed elevated levels of FGF li- 
Fig. 4. Gene expression profile of Fgf ligands in pN60 frontal and parietal bones. a Similarly to what was observed on E17.5 and pN1, Fgf-2 transcript was higher in frontal bone. b RT-PCR analysis did not detected any $F g f-9$ transcript either in frontal or parietal bones. c Fgf-18 expression was detected only in frontal bone. d Increased levels of Runx2 were detected in the frontal bone. Histograms represent the densitometry analysis of RT-PCR products performed using the Image $\mathrm{J}$ software, the relative intensities of bands were normalized to their respective loading control and set as $100 \%$. -rt is a negative control for the reverse transcription reaction performed on pooled RNAs purified from each tissue. The results are presented as the means \pm SD of 3 independent experiments performed on different littermates. ${ }^{*} \mathrm{p}<0.01$. $\mathrm{F}=$ Frontal; $\mathrm{P}=\mathrm{pa}-$ rietal.

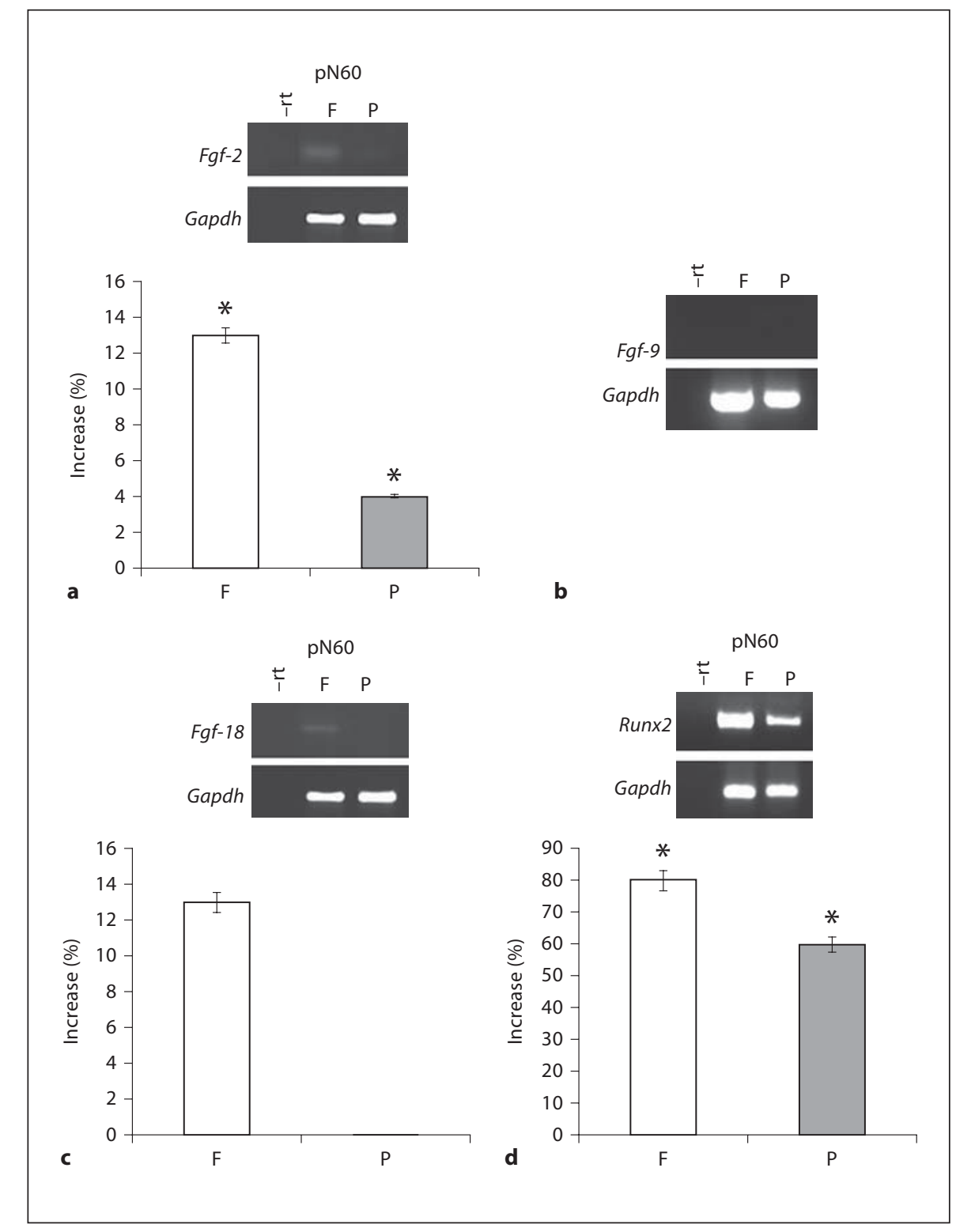

gands compared to the mesoderm-derived parietal bone, we decided to investigate whether there were also regional differences in the expression of FgfRs. Again, fresh tissue was harvested from the frontal and parietal bones of E17.5 mice. RT-PCR analysis demonstrated elevated levels of $F g f R 1, F g f R 2$ and $F g f R 3$ in frontal bone relative to parietal bone, paralleling observed differences in $\mathrm{Fgf}-2$, $F g f-9$ and $F g f-18$ expression in frontal and parietal bone (fig. 5a-c). The expression of $F g f R 2 c$ isoform was much more abundant than that of $F g f R 2 b$. In addition, absence of FgfR4 transcript was observed in both frontal and pa- rietal bones at all time points analyzed (data not shown). The above results were further confirmed by quantitative real-time PCR analysis (online supplementary fig. 1, www.karger.com/doi/10.1159/000202789).

\section{Expression of FgfRs in Newborn Skull Vault}

In frontal and parietal bones derived from $\mathrm{pN} 1$ mice the expression of $F g f R 1$ and $F g f R 3$ genes was similar to that observed in embryonic bones, both transcripts were upregulated in the frontal bone relative to parietal bone (fig. 6a, c). Elevated levels of FgfR $2 b$ isoform were detect- 


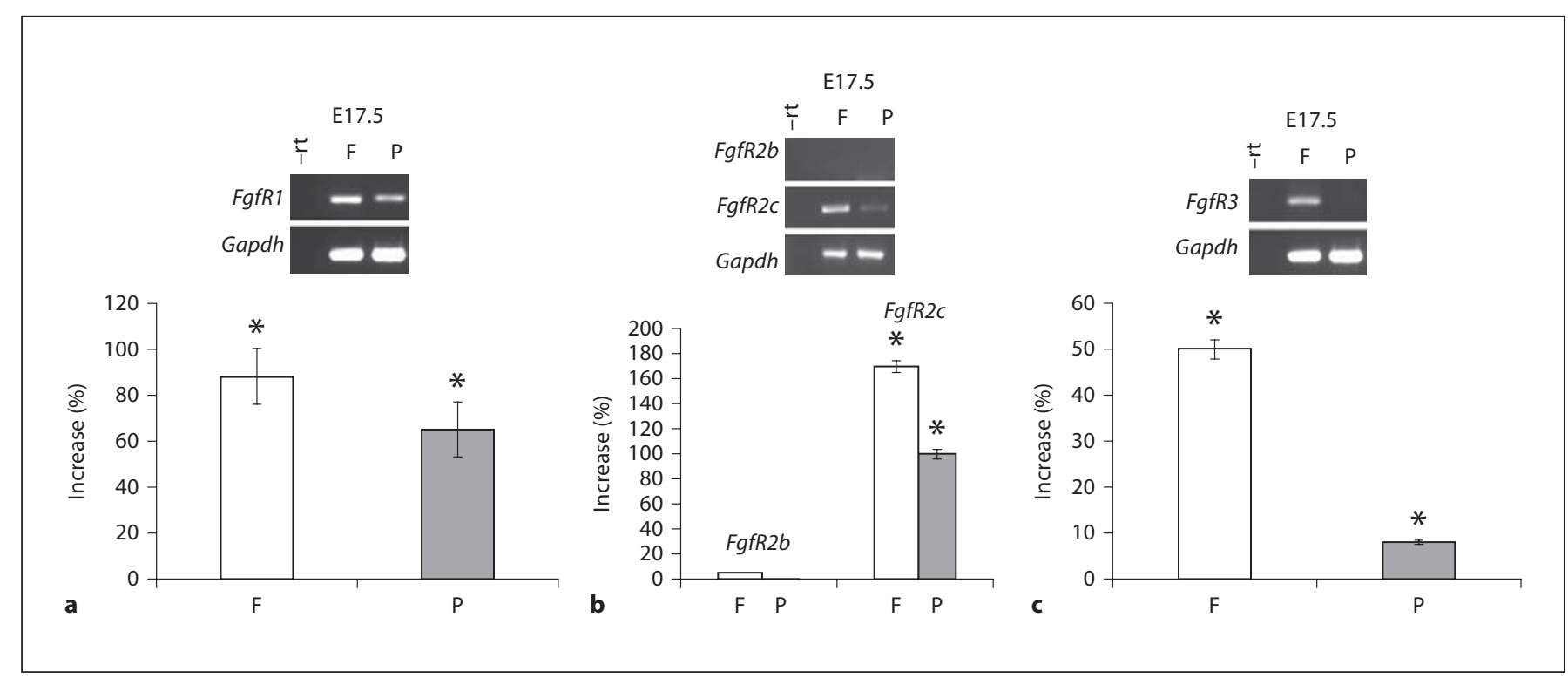

Fig. 5. Gene expression profile of Fgf receptors in E17.5 frontal and parietal bones. a FgfR1 expression analysis by RT-PCR in frontal and parietal bone of E17.5 mice revealed upregulation of $F g f R 1$ transcript in frontal bone. b $F g f R 2 c$ isoform was also strongly upregulated in frontal bone compared to parietal. In contrast, $F g f R 2 b$ isoform was barely detected only in the frontal bone. c Frontal bone elaborates elevated levels of $F g f R 3$ transcripts as well. Histograms represent the densitometry analysis of RT-PCR products performed using the Image J software, the relative intensities of bands were normalized to their respective loading control and set as $100 \%$. - rt is a negative control for the reverse transcription reaction performed on pooled RNAs purified from each tissues. The results are presented as the means $\pm \mathrm{SD}$ of 3 independent experiments performed on different littermates. ${ }^{*} \mathrm{p}<0.01 . \mathrm{F}=$ Frontal; $\mathrm{P}=$ parietal. ed in the frontal bone, while similar levels of $\mathrm{FgfR} 2 \mathrm{c}$ isoform were observed in both frontal and parietal bones (fig. 6b). The above results were further confirmed by quantitative real-time PCR analysis (online supplementary fig. 1, www.karger.com/doi/10.1159/000202789).

\section{Expression of FgfRs in Adult Skull Vault}

A unique expression pattern was observed for FgfR1 gene in pN60 mice. In contrast with what was observed in $\mathrm{E} 17.5$ and $\mathrm{pN} 1$ mice, $\mathrm{pN} 60$ mice expressed higher levels of Fgfrl transcript in the parietal rather than in the frontal bone (fig. 7a). In contrast, both $F g f R 2$ isoforms were upregulated in the frontal bone (fig. $7 \mathrm{~b}$ ). Moreover, in parietal bone the expression level of $\mathrm{Fg} R \mathrm{R} 2 \mathrm{c}$ isoform was markedly lower compared to that of $\mathrm{pN} 1$ parietal bone. Frontal bone also elaborated elevated levels of $F g f R 3$ transcripts versus parietal bone (fig. 7c). The above results were further confirmed by quantitative real-time PCR analysis (online supplementary fig. 1, www.karger.com/doi/10.1159/000202789).

\section{Differential Cell Proliferation Activity of Frontal and Parietal Bones}

The higher expression of Runx2 observed in frontal bone compared to parietal bone would suggest the presence of a larger pool of osteoprogenitor cells in frontal bone. Because osteoprogenitor cells are less differentiated and therefore more proliferative, we reasoned to analyze the proliferation activity of frontal and parietal primary osteoblasts harvested from E17.5, pN1 and pN60 mice. Proliferation was evaluated by BrdU incorporation. After $24 \mathrm{~h}$, osteoblasts of frontal bone origin demonstrated significantly greater incorporation of BrdU than osteoblasts of parietal origin (fig. 8a). Thus, frontal bone-derived osteoblasts from E17.5, pN1 and pN60 mice have superior proliferative activity than those from parietal bone $(\mathrm{p}<$ 0.01 ). In addition, immunohistochemistry analysis performed on frontal and parietal bones using anti-PCNA antibody also detected more proliferative osteoblasts in frontal than parietal bone (fig. 8b). Furthermore, PCNA staining was also observed in the periostium and dura mater of both, frontal and parietal bones of $\mathrm{pN} 1$ mice, as well as in the periostium of frontal bone of pN60 mice. 


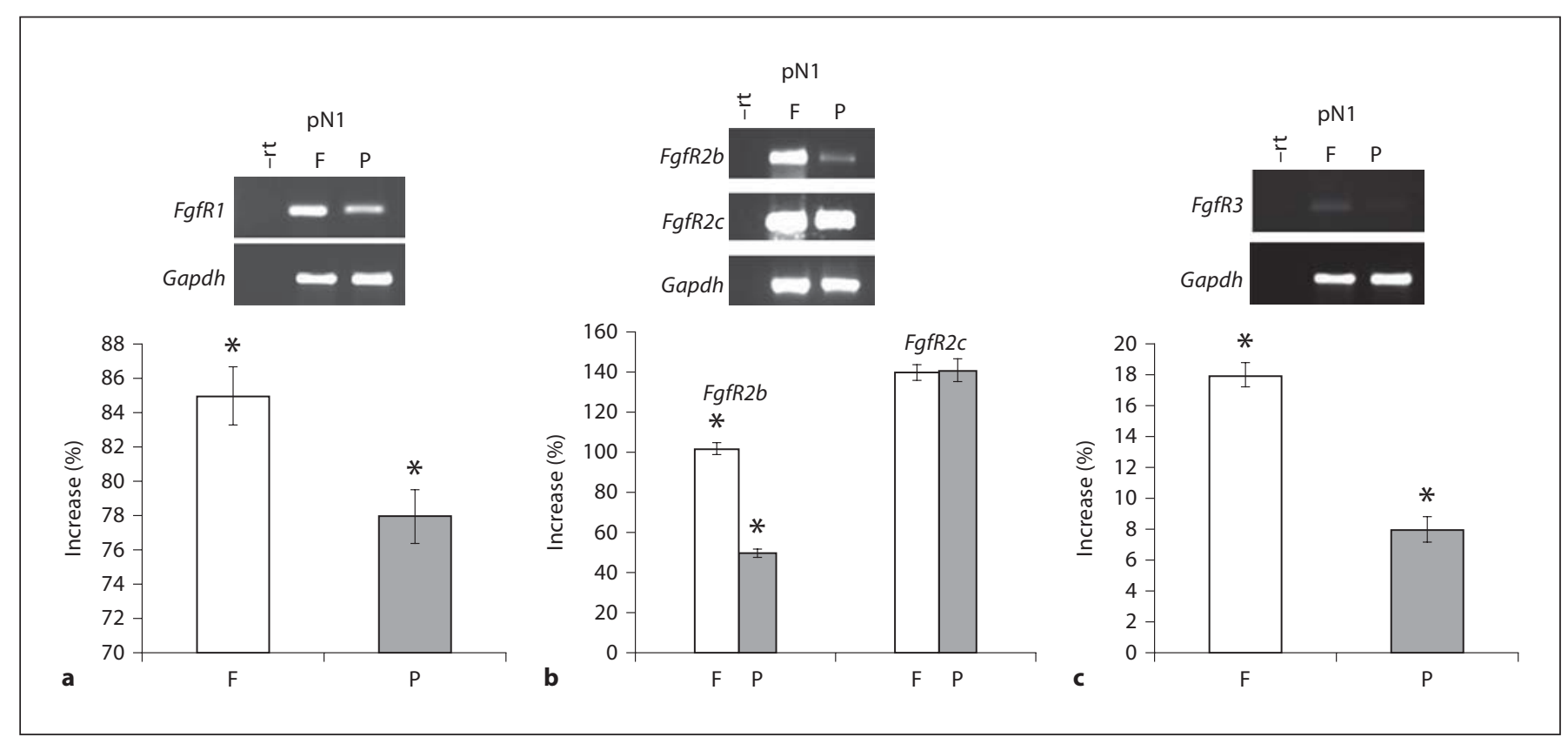

Fig. 6. Gene expression profile of Fgf receptors in pN1 frontal and parietal bones. a Frontal bone elaborates higher levels of FgfR1 transcript in pN1 mice. b FgfR $2 b$ is upregulated in frontal bone, while $F g f R 2 c$ is expressed at similar levels in both frontal and parietal bones. c A faint expression of $F g f R 3$ was detected in $\mathrm{pN} 1$ frontal and parietal bone, with higher levels in frontal bone. Histograms represent the densitometry analysis of RT-PCR products performed using the Image J software, the relative intensities of bands were normalized to their respective loading control and set as $100 \%$. $-\mathrm{rt}$ is a negative control for the reverse transcription reaction performed on pooled RNAs purified from each tissue. The results are presented as the means \pm SD of 3 independent experiments performed on different littermates. ${ }^{*} \mathrm{p}<0.01 . \mathrm{F}=$ Frontal; $\mathrm{P}=$ parietal.

\section{Discussion}

The mammalian skull vault has a mixed developmental tissue origin that reflects its heterogenous evolutionary origin. Indeed, this complex history has functional consequences for the pattern of skull vault growth, and for the genetic control of the tissue interactions that are responsible for maintaining a balance between osteogenic cell proliferation and differentiation. Studies using a transgenic mouse with a permanent neural crest cell lineage marker, Wnt1-Cre/R26R, have revealed that the frontal bones are neural crest derived and the parietal bones mesodermal.

The present study is a characterization of frontal and parietal bones at their molecular level. In order to define potential differences between these 2 calvarial bones, we have analyzed their gene expression pattern for the main Fgfligands and receptors known to be important for the osteogenic differentiation program. The importance of FGF/FGFR signaling in human skull development has been revealed by the identification of mutations in FGFR genes in several craniosynostoses [Wilkie et al., 1995a, b] as well as by transgenic mouse models [Coffin et al., 1995; Deng et al., 1996; Eswarakumar et al., 2002; Liu et al., 2002; Ohbayashi et al., 2002; Govindarajan and Overbeek, 2006; Hung et al., 2007].

Taken together, the data gathered from this study strongly suggest that tissue origin translates in marked molecular differences between the neural crest-derived frontal and the mesodermal-derived parietal bone in terms of the expression levels of $\mathrm{Fgfs} / \mathrm{Fg} f R s$ genes. From embryonic stages (E17.5) through newborn and adult mice, frontal bone elaborates higher levels of $F g f s / F g f R s$ transcripts versus parietal bone. This observation is interesting and would suggest that the frontal bone is a domain with higher FGF-signaling competence than parietal bone. Our analysis also revealed that Runx-2 expression is higher in frontal bone compared to parietal bone at all time points investigated. The higher expression of Runx2 may reflect the presence of a larger pool of osteoprogenitor cells [Ducy et al., 1997] in the frontal bone due to the elevated levels of Fgf ligands. Indeed, the higher 


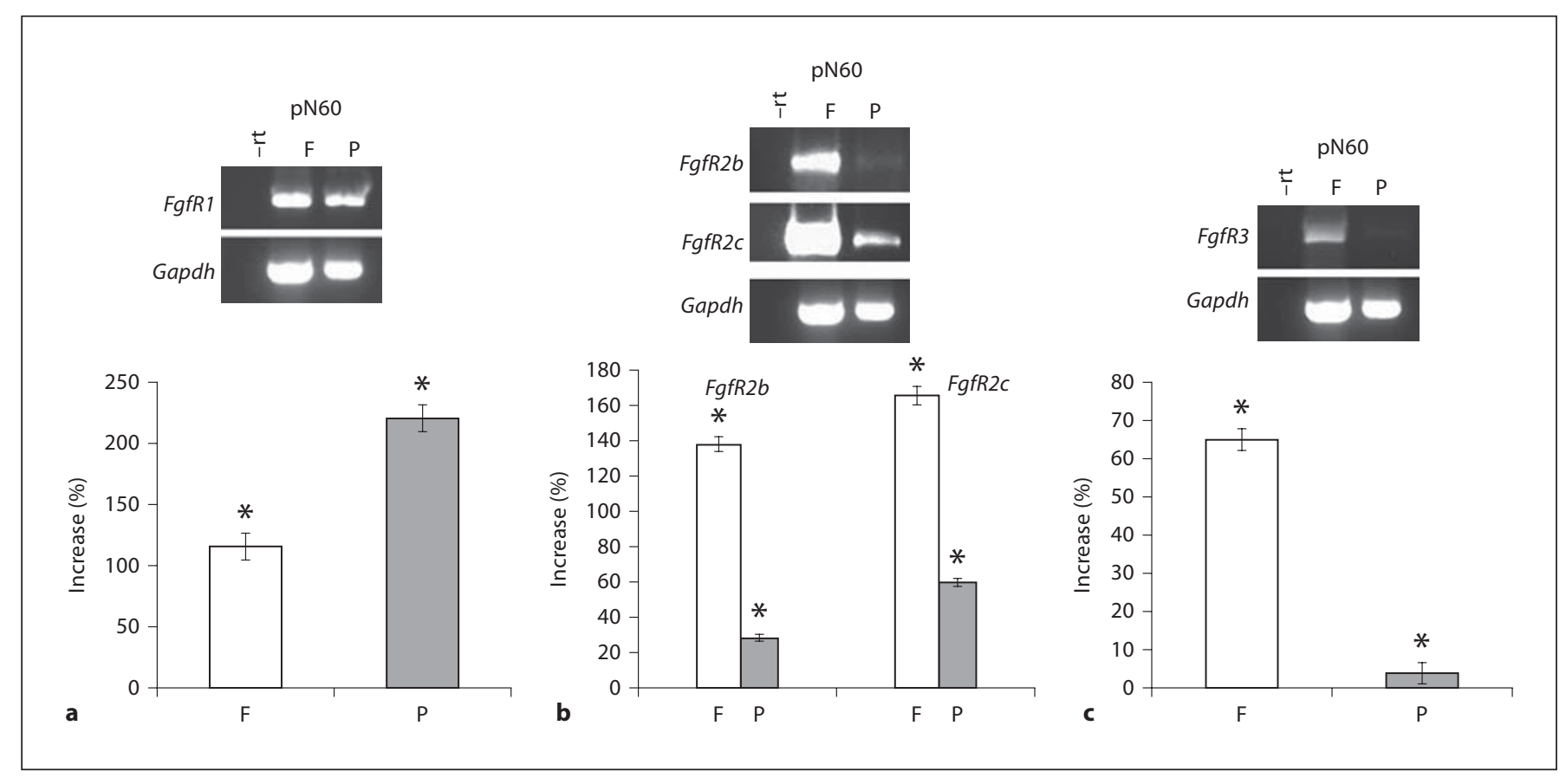

Fig. 7. Gene expression profile of Fgf receptors in pN60 frontal and parietal bones. a RT-PCR analysis shows that FgfR 1 was higher in parietal bone. b Elevated levels of both $F g f R 2 b$ and $F g f R 2 c$ were detected in frontal bone, whereas much lower levels of transcripts were observed in parietal bone. c High levels of FgfR3 gene expression were also detected in the frontal bone of pN60 mice. Histograms represent the densitometry analysis of RT-PCR prod- ucts performed using the Image J software, the relative intensities of bands were normalized to their respective loading control and set as $100 \%$. $-r t$ is a negative control for the reverse transcription reaction performed on pooled RNAs purified from each tissue. The results are presented as the means \pm SD of 3 independent experiments performed on different littermates ${ }^{*} \mathrm{p}<0.01 . \mathrm{F}=$ Frontal; $\mathrm{P}=$ parietal. proliferation activity observed in frontal bone-derived osteoblasts further supports this hypothesis. Several studies have indicated that Fgf ligands, such as Fgf-2 and Fgf- 9 , act as positive regulators of osteoprogenitor cells by triggering their proliferation, and therefore enriching a pool of osteoprogenitor cells [Kim et al., 2003; Fakhry et al., 2005; Quarto and Longaker, 2006]. The above observation leads us to speculate that the frontal bone may be endowed with higher potential for tissue repair due to the presence of a larger number of putative osteoprogenitor cells. This hypothesis is currently under investigation [Quarto et al., in preparation].

Mirroring the expression pattern of $\mathrm{Fg} f$ ligands, frontal bone also elaborates higher levels of $F g f R$ transcripts than parietal bone. However, among the 3 stages analyzed, differences in the expression of $F g f R 1$ gene profile were observed. Adult, pN60 mice elaborated higher levels of transcript in the parietal bone, while E17.5 and pN1 mice expressed more $F g f R 1$ transcripts in the frontal bone.

FGF/FGFR Genes Profiling in Different Tissue-Derived Skull Bones
Because our data indicated a consistent upregulation of Fgf-2, Fgf-9 and Fgf-18 as well as FgfR1, FgfR2 and $F g f R 3$ genes expression in the neural crest-derived frontal bone, we reasoned to investigate whether the expression of other important osteogenic genes such as Bmp ligands and their receptors mirror that of $F g f / F g f R s$ in frontal and parietal bones. A preliminary analysis of Bmp-2 and Bmp-4, as well as receptors BmpRIA and $B m p R I B$ indicated that the expression pattern of these osteogenic molecules in the frontal and parietal bones does not mirror that of $F g f / F g f R s$. Bmp-2 was the only gene upregulated exclusively in frontal bone derived from pN1 mice, while elevated levels of BmpRIA transcript were detected in the parietal bone of pN60 mice. Overall no significant quantitative differences in the expression pattern of $B m p-4$ and $B m p R I B$ were observed between the frontal and parietal bones in E17.5, pN1 and pN60 mice (data not shown). Thus, the quantitative differential gene expression observed between the frontal and parietal bones appears unique to $F g f / F g f R s$.

Cells Tissues Organs 2009;190:158-169 


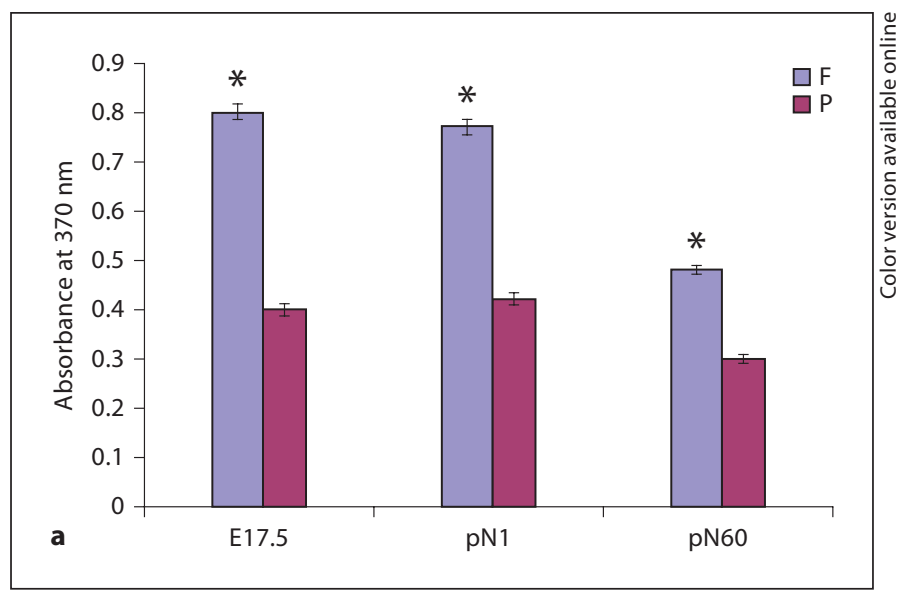

Fig. 8. Proliferation of frontal bone and parietal bone derived osteoblasts. a Cell proliferation of frontal and parietal osteoblasts was assessed by BrdU incorporation at $24 \mathrm{~h}$. Frontal osteoblasts proliferated greater than those of parietal bone origin. Results are means \pm SD of 3 independent experiments. ${ }^{*} \mathrm{p}<0.01$. b Immunolocalization of PCNA protein on frontal and parietal bones using a specific anti-PCNA antibody. More PCNA-positive osteoblasts (arrows) were present in frontal bone versus parietal bone. Intense PCNA staining was also present in periostium and dura mater cells. $\mathrm{F}=$ Frontal; $\mathrm{P}=$ parietal; $\mathrm{Pr}=$ periostium; $\mathrm{Ob}=$ osteoblasts; $\mathrm{Dm}=$ dura mater.

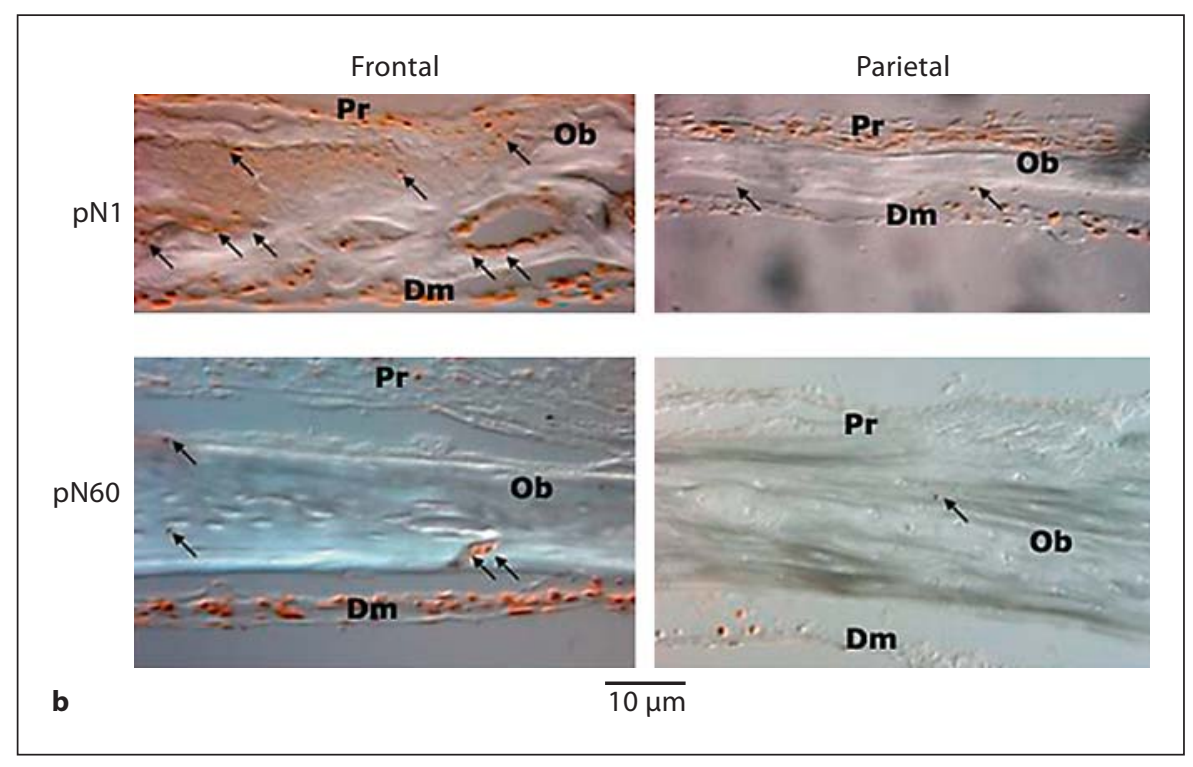

Previous studies performed by in situ hybridization have mainly focused on the expression pattern of $F g f R$ genes during craniofacial bone development and cranial suture patterning in embryonic animals [Iseki et al., 1999; Rice et al., 2003]. To our knowledge, the study reported here represents the first comparative and quantitative analysis of the expression pattern of osteogenic $\mathrm{Fg} f \mathrm{~S} /$ FgfRs genes, performed focusing on frontal and parietal bones in embryonic, newborn and adult mice. This study highlights distinct and sustained differences in the expression pattern of $F g f s / F g f R s$ genes between the 2 bones of different tissue origin. Indeed, the data suggest that embryonic tissue origin differences determine a distinct FGF-signaling pattern in frontal and parietal bones, and that the neural crest-derived frontal bone represents a more competent domain for the FGF signaling.

\section{Acknowledgments}

This work was supported by NIH grants R01DE13194 and R01DE14526 to M.T.L. and by the Oak Foundation.

References

Chai, Y., X. Jiang, Y. Ito, P. Bringas Jr., J. Han, D.H. Rowitch, P. Soriano, A.P. McMahon, H.M. Sucov (2000) Fate of the mammalian cranial neural crest during tooth and mandibular morphogenesis. Development 127: 1671-1679.

Coffin, J.D., R.Z. Florkiewicz, J. Neumann, T. Mort-Hopkins, G.W. Dorn 2nd, P. Lightfoot, R. German, P.N. Howles, A. Kier, B.A. O'Toole, et al. (1995) Abnormal bone growth and selective translational regulation in basic fibroblast growth factor (FGF-2) transgenic mice. Mol Biol Cell 6: 1861-1873. 
Deng, C., A. Wynshaw-Boris, F. Zhou, A. Kuo, P. Leder (1996) Fibroblast growth factor receptor 3 is a negative regulator of bone growth. Cell 84: 911-921.

Ducy, P., R. Zhang, V. Geoffroy, A.L. Ridall, G. Karsenty (1997) Osf2/Cbfa1: a transcriptional activator of osteoblast differentiation. Cell 89: 747-754.

-Eswarakumar, V.P., E. Monsonego-Ornan, M. Pines, I. Antonopoulou, G.M. Morriss-Kay, P. Lonai (2002) The IIIc alternative of Fgfr2 is a positive regulator of bone formation. Development 129: 3783-3793.

Fakhry, A., C. Ratisoontorn, C. Vedhachalam, I. Salhab, E. Koyama, P. Leboy, M. Pacifici, R.E. Kirschner, H.D. Nah (2005) Effects of FGF-2/-9 in calvarial bone cell cultures: differentiation stage-dependent mitogenic effect, inverse regulation of BMP-2 and noggin, and enhancement of osteogenic potential. Bone 36: 254-266.

Govindarajan, V., P.A. Overbeek (2006) FGF9 can induce endochondral ossification in cranial mesenchyme. BMC Dev Biol 6: 7 .

-Hung, I.H., K. Yu, K.J. Lavine, D.M. Ornitz (2007) FGF9 regulates early hypertrophic chondrocyte differentiation and skeletal vascularization in the developing stylopod. Dev Biol 307: 300-313.

Iseki, S., A.O. Wilkie, G.M. Morriss-Kay (1999) Fgfrl and Fgfr2 have distinct differentiationand proliferation-related roles in the developing mouse skull vault. Development 126: 5611-5620.
Jiang, X., S. Iseki, R.E. Maxson, H.M. Sucov, G. M. Morriss-Kay (2002) Tissue origins and interactions in the mammalian skull vault. Dev Biol 241: 106-116.

Karsenty G. (2001) Minireview: transcriptional control of osteoblast differentiation. Endocrinology 142: 2731-2733.

Karsenty, G., E.F. Wagner (2002) Reaching a genetic and molecular understanding of skeletal development. Dev Cell 2: 389-406.

Kim, H.J., J.H. Kim, S.C. Bae, J.Y. Choi, H.J. Kim, H.M. Ryoo (2003) The protein kinase C pathway plays a central role in the fibroblast growth factor-stimulated expression and transactivation activity of Runx2. J Biol Chem 278: 319-326.

Liu, Z., J. Xu, J.S. Colvin, D.M. Ornitz (2002) Coordination of chondrogenesis and osteogenesis by fibroblast growth factor 18 . Genes Dev 16: 859-869.

Mansukhani, A., P. Bellosta, M. Sahni, C. Basilico (2000) Signaling by fibroblast growth factors (FGF) and fibroblast growth factor receptor 2 (FGFR2)-activating mutations blocks mineralization and induces apoptosis in osteoblasts. J Cell Biol 149: 1297-1308.

Marie, P.J. (2003) Fibroblast growth factor signaling controlling osteoblast differentiation. Gene 316: 23-32.

Morriss-Kay, G.M. (2001) Derivation of the mammalian skull vault. J Anat 199: 143151.

Muenke, M., U. Schell (1995) Fibroblast-growthfactor receptor mutations in human skeletal disorders. Trends Genet 11: 308-313.
Naski, M.C., D.M. Ornitz (1998) FGF signaling in skeletal development. Front Biosci 3: d781-d794.

Dhbayashi, N., M. Shibayama, Y. Kurotaki, M. Imanishi, T. Fujimori, N. Itoh, S. Takada (2002) FGF18 is required for normal cell proliferation and differentiation during osteogenesis and chondrogenesis. Genes Dev 16: $870-879$.

Ornitz, D.M., N. Itoh (2001) Fibroblast growth factors. Genome Biol 2: REVIEWS3005.

Quarto, N., K.D. Fong, M.T. Longaker (2005) Gene profiling of cells expressing different FGF-2 forms. Gene 356: 49-68.

Quarto N., M.T. Longaker (2006) FGF-2 inhibits osteogenesis in mouse adipose tissue-derived stromal cells and sustains their proliferative and osteogenic potential state. Tissue Eng 12: 1405-1418.

-Rice, D.P., R. Rice, I. Thesleff (2003) Fgfr mRNA isoforms in craniofacial bone development. Bone 33: 14-27.

Wilkie A.O., G.M. Morriss-Kay, E.Y. Jones, J.K. Heath (1995a) Functions of fibroblast growth factors and their receptors. Curr Biol 5: 500507.

Wilkie A.O., S.F. Slaney, M. Oldridge, M.D. Poole, G.J. Ashworth, A.D. Hockley, R.D. Hayward, D.J. David, L.J. Pulleyn, P. Rutland, et al., (1995b) Apert syndrome results from localized mutations of FGFR2 and is allelic with Crouzon syndrome. Nat Genet 9: 165-172. 